\title{
Diagnostics of the Solar Dynamo Using the Observed Pattern of Surface Magnetic Fields
}

\author{
J.O. Stenflo \\ Institute of Astronomy, ETH-Zentrum, CH-8092 Zurich, Switzerland
}

\begin{abstract}
The solar surface represents for the solar dynamo an outer boundary that is directly accessible to observations. The evolutionary and rotational properties of the magnetic fields at this boundary can be empirically determined using existing synoptic magnetograph data. The derived properties provide detailed constraints on the underlying theory, such that an inversion approach to the dynamo problem becomes feasible. Ambiguities in the interpretation may be removed using the independent constraints from helioseismology.
\end{abstract}

\section{Introduction}

Most solar dynamo work of the past has aimed at qualitatively reproducing the butterfly diagram of sunspots. The sunspots are however only secondary manifestations of the primary dynamo parameter, the magnetic field, they reflect only a certain aspect of the magnetic field (intense concentrations of toroidai flux), and they occur only at certain discrete locations on the sun. The magnetic fields, on the other hand, have been observed over the whole solar disk for three decades now, since 1959, and provide us with rich empirical constraints that may be used for dynamo inversions. The detailed magnetograph data however need to be converted into forms more suitable for direct diagnostic purposes, as will be described in Sects. 2-4.

Besides revealing the evolutionary properties and global wave modes of the solar dynamo, the surface magnetic flux can also be used as a tracer for determinations of the solar rotation. The rotational phase velocity of the magnetic field pattern depends however not only on processes in the surface layers, but also on the processes in the deep layers of the solar interior, from which the surface fields are being replenished. In Sect. 5 we will indicate how these various effects may be untangled. 


\section{Data base}

Magnetograms (maps of the line-of-sight component of the magnetic field) have been recorded daily at the Mount Wilson Observatory since August 1959 and at the Kitt Peak Observatory since 1976. We do not have information on the vector magnetic field, but for investigations of the evolution of the field pattern it is a good approximation to assume that the true orientation of the field is on the average in the radial direction in the layers, where the field is being measured (the photosphere). The justification for this approximation is that the photospheric magnetic flux is extremely fragmented in individual fluxtubes, and the buoyancy forces acting on these fluxtubes in the radial direction are very strong due to the rapid exponential density decrease in the photosphere. The magnetograms may then be converted into maps of the radial component of the magnetic flux. When we in the following talk about the surface magnetic field $B$, we mean the radial magnetic flux, although we omit index $r$ for brevity of notation.

For a global mode analysis we like to expand the surface magnetic field pattern in the spherical harmonics $Y_{\ell}^{m}(\vartheta, \varphi)$, where $\vartheta$ is the colatitude, $\varphi$ the longitude:

$$
B(\vartheta, \varphi, t)=\sum_{\ell=0}^{\infty} \sum_{m=-\ell}^{\ell} c_{\ell}^{m}(t) Y_{\ell}^{m}(\vartheta, \varphi) .
$$

The dependence of $B$ on time $t$ is then accounted for by the time dependence of the complex harmonic coefficients $c_{\ell}^{m}$. Using the orthonormal properties of the spherical harmonics, we can solve for the coefficients:

$$
c_{\ell}^{m}(t)=\int B(\vartheta, \varphi, t) Y_{\ell}^{m *}(\vartheta, \varphi) \mathrm{d} \Omega
$$

The harmonic decomposition cannot however be carried out entirely as described by Eqs. (1) and (2), since the sun is not observed from all sides at the same time. Observational sampling of the magnetic field over all longitudes can only be accomplished over the course of one solar rotation, i.e., about 27 days. Since the magnetic field pattern evolves significantly over this period, the longitude $\varphi$ and time $t$ coordinates are no longer independent of each other. Moreover, as the sun rotates differentially, there is no unique way to define a longitude system on the sun (since any longitude system rotates rigidly). We will return to these questions later.

A condensed synoptic data set that is easier to work with can be extracted from the full-disk daily magnetograms by sampling the magnetic field in one longitude band around the central meridian in each magnetogram. As the sun rotates, different longitudes (decreasing from $360^{\circ}$ to $0^{\circ}$ ) pass the central meridian, so during one month all longitudes get sampled, and a synoptic map can be constructed, giving the radial magnetic field over all latitudes and longitudes of the sun. The longitude system normally adopted for such maps is based on the Carrington rotation period $P_{C}=27.2753$ days. Let us denote the corresponding rotation frequency by $\nu_{C}=1 / P_{C}$. Then we have the following relation between the Carrington longitude $\varphi$ and time $t$ : 


$$
\varphi=-2 \pi\left(\nu_{C} t-n\right) \text {. }
$$

$n$ is an integer that ensures that $\varphi$ always stays in the interval $-\pi<\varphi \leq \pi$. For convenience we have chosen the zero point of the time scale such that $\varphi=0$ when $t=0$.

The choice of the longitude system is arbitrary and does not affect the analysis that will be described in the rest of the present review. If we want we can always replace the longitude coordinate $\varphi$ by time $t$, using $\mathrm{Eq}$. (3), and consider the magnetic field $B$ as a function of latitude and time only. It is also useful to replace the colatitude $\vartheta$ by $x=\cos \vartheta$, since $x$ represents the projected distance on the sun (when the axis of rotation is perpendicular to the line of sight), and the synoptic maps are digitized at points equidistant in $x$. The magnetic field is thus basically a function of $x$ and $t$, and may be written $B(x, t)$.

The Kitt Peak data have been recorded with much higher spatial resolution than the Mount Wilson data. To obtain a homogeneous data base extending all the way back to 1959 we have therefore spatially averaged the Kitt Peak data and used them from 1976 onwards. The selected common format for the whole synoptic data set is defined by a grid of 30 zones equidistant in $x$ (the sine of the latitude), and 36 sectors equidistant in longitude. In the following we will describe the results based on such a synoptic data base extending over a period of $26 \mathrm{yr}$.

\section{Evolution of the axisymmetric field component}

\section{1 "Butterfiy diagram" of the radial magnetic flux}

The axisymmetric component of the magnetic field pattern may be obtained by averaging over all longitudes:

$$
\bar{B}(x, t)=\frac{1}{2 \pi} \int_{-\pi}^{\pi} B(x, \varphi, t) \mathrm{d} \varphi .
$$

This longitude averaging may also be regarded as a smoothing of the data using a rectangular time window with a width of one Carrington rotation. The results of this smoothing process are not sensitive to the exact choice of width for the time window, except that a wider window is useful to suppress noise and short-term fluctuations. For the harmonic analysis we will use a window of $1 P_{C}$ as in Eq. (4), but for a smoother representation of "butterfly diagrams" we will apply a time window of $16 P_{C}$ (corresponding to $1.2 \mathrm{yr}$ ).

The time smoothed $\bar{B}$ can be graphically represented as isocontours in latitude - time space, analogous to the representation of sunspots in the form of "butterfly diagrams". Figure 1 gives $\vec{B}$, smoothed with a running $16 P_{C}$ window, vs. $x$ (sine of the latitude) and time $t$. The pattern is clearly predominantly of odd parity, i.e., anti-symmetric with respect to reflections in the equatorial plane. It exhibits the well-known features of polarity reversals every $11 \mathrm{yr}$, equatorward migration of the low-latitude zones, and steep poleward migration of the higher-latitude zones. 


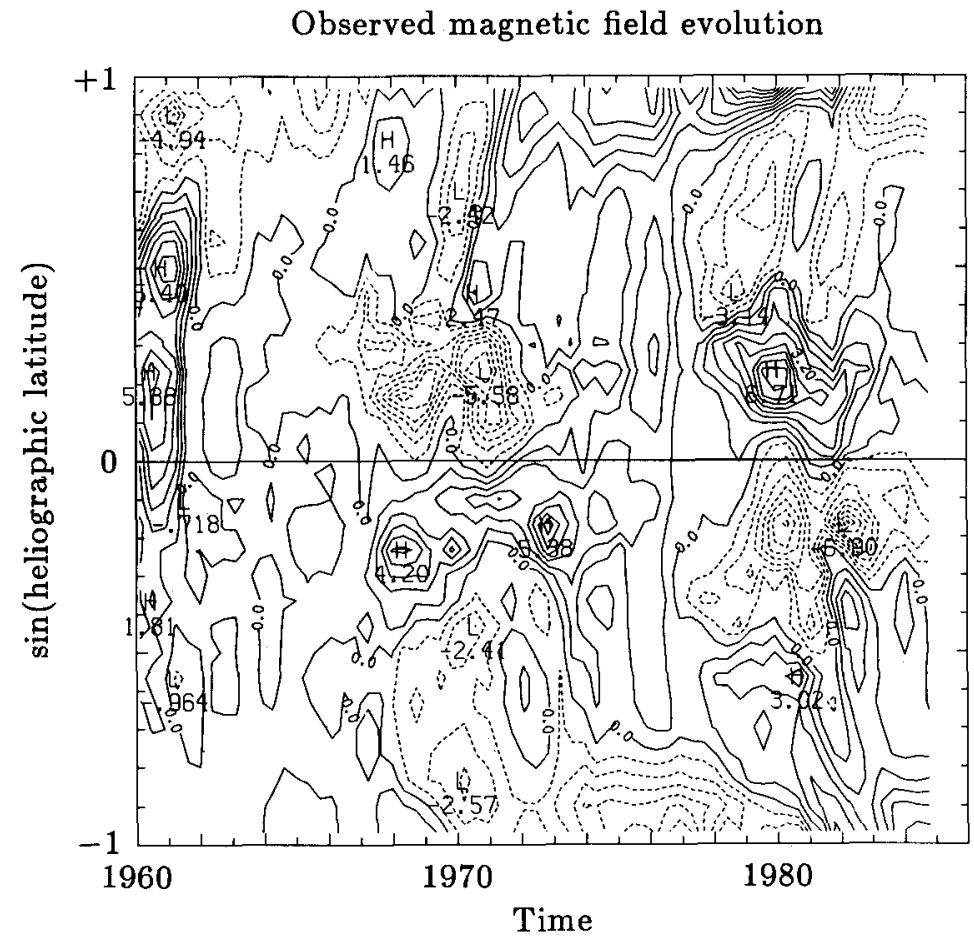

Figure 1. Isocontours of the axially symmetric component of the radial magnetic field, vs. time and sine of the latitude. The solid (dashed) contours represent fields of positive (negative) polarity. From Stenflo (1988).

\subsection{Power spectrum analysis of the axisymmetric modes}

We can explore whether the pattern of Fig. 1 can be explained in terms of globally resonant waves by making a harmonic decomposition of the pattern and a power spectrum analysis of the harmonic coefficients. The power spectrum $P c_{\ell}^{m}(\nu)$ is given by

$$
P c_{\ell}^{m}(\nu)=\frac{1}{T_{e}}\left|\tilde{c}_{\ell}^{m}(\nu)\right|^{2},
$$

where $T_{e}$ is the effective length of the time series (taking apodization and data gaps into account), and

$$
\tilde{c}_{\ell}^{m}(\nu)=\int_{-\infty}^{\infty} c_{\ell}^{m}(t) e^{-i 2 \pi \nu t} \mathrm{~d} t
$$

is the Fourier transform of the harmonic coefficients. The axisymmetric harmonic coefficients (with $m=0$ ) are obtained from the Legendre transform of $\bar{B}$, as follows from Eqs. (2) and (4):

$$
c_{\ell}^{0}(t)=\sqrt{\pi(2 \ell+1)} \int_{-1}^{1} \bar{B}(x, t) P_{\ell}(x) \mathrm{d} x
$$




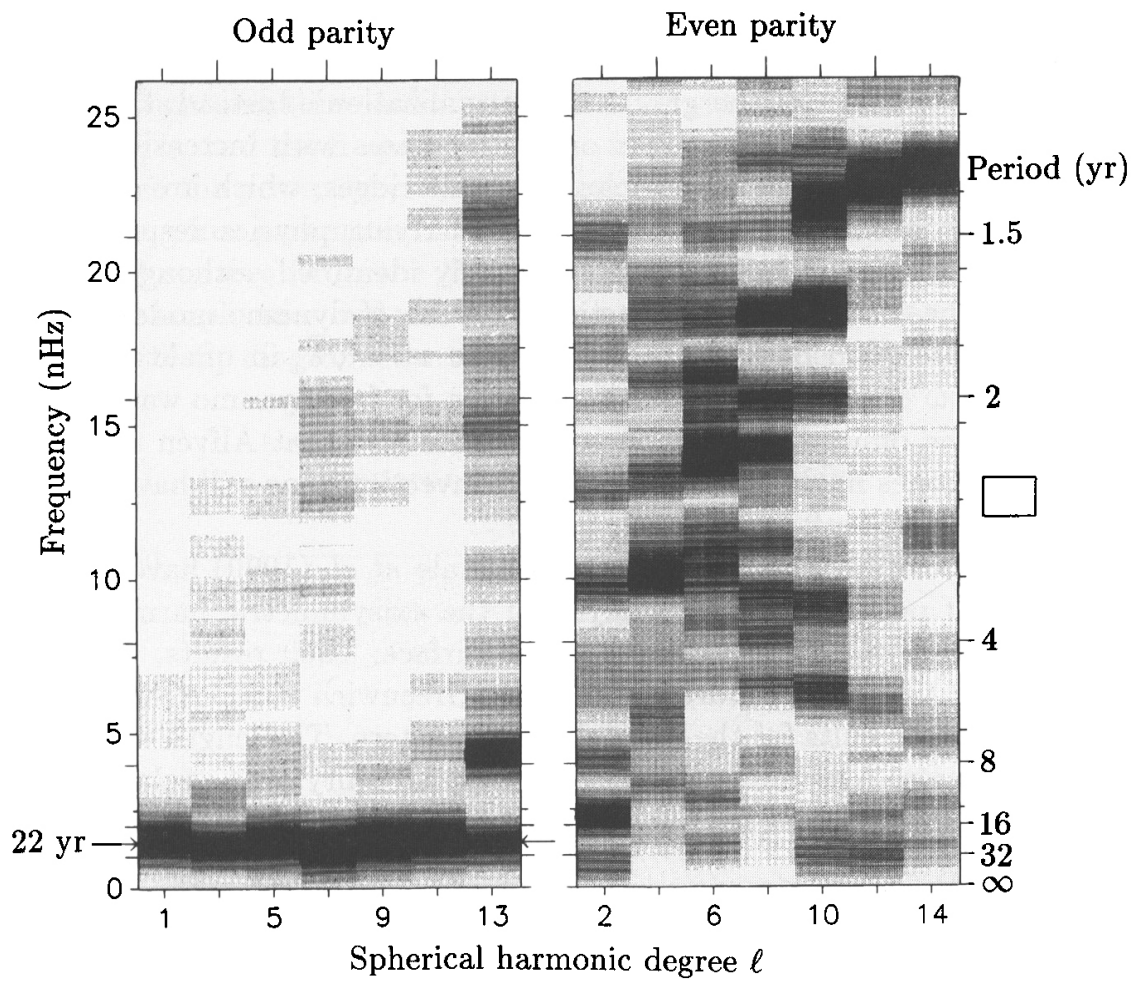

Figure 2. Power spectra of the axisymmetric harmonic coefficients of the radial magnetic field, as functions of frequency and $\ell$. The white areas correspond to zero power, the darkest areas to the maximum spectral density for the given $\ell$ value. The resolution is indicated by the small box to the right of the diagram. From Stenflo (1988).

Power spectra $P c_{\ell}^{0}(\nu)$ for the axisymmetric modes were first obtained by Stenflo and Vogel (1986). Figure 2 shows $P c_{\ell}^{0}$ for the present 26 year data set. Odd and even parity refer to odd and even values of $\ell$, and correspond to patterns that are anti-symmetric and symmetric, respectively, with respect to reflections in the equatorial plane.

We see that the power is not distributed continuously over the diagram, but is strongly concentrated around one frequency for each value of $\ell$. The power amplitudes of the even modes are 5-10 times smaller as compared with the odd modes, but they have been made visible by normalizing the gray scale in Fig. 2 independently for each $\ell$ value to the maximum power in the respective $\ell$ band. The low amplitudes of the even modes explain why the background noise is more prominent in the even-parity diagram as compared with the odd-parity one.

The most striking feature of Fig. 2 is the entirely different behaviour of the odd and even modes, giving evidence for the operation of a strict parity selection rule on the sun. For the odd modes the power is concentrated around the $22 \mathrm{yr}$ resonance, with hardly any sign of a second harmonic at $11 \mathrm{yr}$. In contrast the even 
modes do not show any trace of the $22 \mathrm{yr}$ resonance (the peak of the quadrupole $\ell=2$ mode occurs at a period of $14 \mathrm{yr}$ and is of very small amplitude - it appears prominent as a result of the gray-scale normalization). Instead, the frequency at which maximum even-mode power occurs increases with increasing $\ell$ value, in a way reminiscent of the helioseismology $\mathrm{p}$-mode ridges, which are due to standing global waves in the sun's interior. The underlying physics responsible for this dispersion relation has not yet been definitely identified, although Hoyng (1987, 1988,1990 ) has shown that stochastic excitation of dynamo modes would lead to a dispersion relation of the type $\omega \sim \sqrt{k}$ or $\nu \sim \sqrt{\ell}$, in qualitative agreement with Fig. 2. $\omega \sim \sqrt{k}$ is the dispersion relation for free dynamo waves in the $\alpha-\omega$ dynamo if one omits the diffusion terms. Note also that Alfvén waves along $k \mathrm{G}$ toroidal fluxropes in the lower part of the convection zone will have periods in the observed range (Stenflo and Vogel, 1986).

Gokhale and Javaraiah (1989) and Gokhale et al. (1990) have performed the same type of power spectrum analysis of the axisymmetric harmonic modes for the distribution of sunspots over the solar surface. Their results, which are based on the more than one hundred years long Greenwich sunspot data set, confirm and extend our results for the magnetic field pattern. The length of the Greenwich data set has also allowed them to explore the stability of the relative amplitudes and phases of the modes of different $\ell$ values when moving in time from cycle to cycle.

\section{3 "Modal cleaning" of the butterfly diagram}

The circumstance that the power spectrum diagram of Fig. 2 has the character of an emission-line spectrum with a weak and noisy continuous background suggests that the sun's magnetic field can to a good approximation be described as a superposition of discrete global modes, which may be the eigenmodes of an underlying linear wave equation.

Assuming that the observed pattern can be represented by one discrete global mode per $\ell$ value, the axisymmetric part of Eq. (1) can be written as

$$
B(x, t)=\operatorname{Re} \sum_{\ell=1}^{N} a_{\ell} e^{i 2 \pi \nu_{\ell} t} P_{\ell}(x),
$$

where $N$ is the maximum $\ell$ value that we have spatially resolved $(N=14$ in our case), and $a_{\ell}$ are the complex mode amplitudes. Eq. (8) implies that we approximate the power spectrum of Fig. 2 as a superposition of $\delta$-function peaks, one for each $\ell$ value.

If we factorize each complex amplitude in an absolute value and a phase factor, Eq. (8) can be written as

$$
B(x, t)=\sum_{\ell=1}^{N}\left|a_{\ell}\right| \cos \left[2 \pi \nu_{\ell}\left(t-t_{\ell}\right)\right] P_{\ell}(x),
$$

where we have introduced a time lag $t_{\ell}$ related to the phase $\phi_{\ell}$ by 


$$
\phi_{\ell}=-2 \pi \nu_{\ell} t_{\ell}
$$

Equation (9) contains three unknowns for each $\ell$ value: the absolute amplitude $\left|a_{\ell}\right|$, the time lag $t_{\ell}$, and the frequency $\nu_{\ell}$. The frequency can be fixed by choosing the value at which the power spectrum maximum occurs for each $\ell$ value in Fig. 2 . The two remaining free parameters for each of the 14 modes can be determined by an iterative least squares fit to the "butterfly diagram" of Fig. 1 (Stenflo, 1988). This gives us the amplitudes and phases of the modes, and allows us to reconstruct "cleaned-up" butterfly diagrams through superposition of selected discrete modes, using Eq. (9).

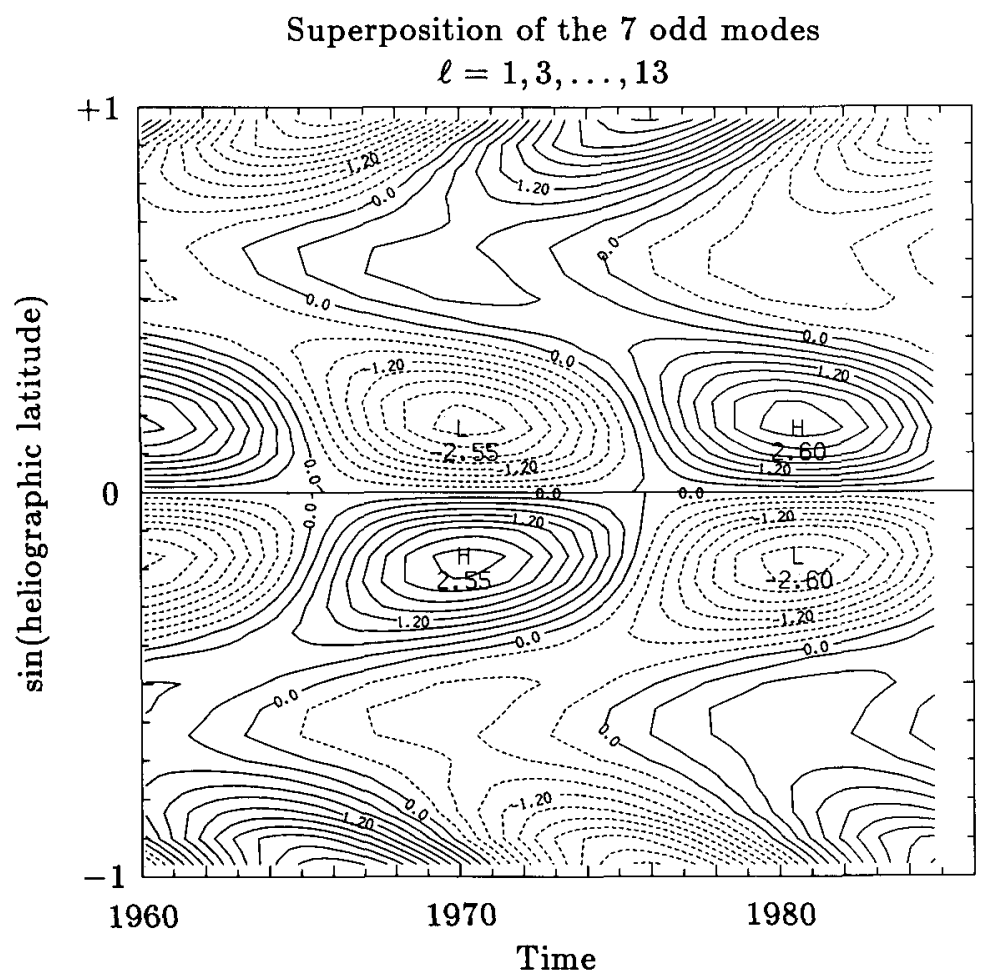

Figure 3. The axisymmetric component of the radial magnetic field described as a superposition of 7 discrete harmonic modes of odd parity, each varying sinusoidally with a 22 yr period. From Stenflo (1988).

As the odd modes are the dominating ones and are all represented by one and the same frequency, $(22 \mathrm{yr})^{-1}$, it is particularly useful to reconstruct the "modally cleaned" butterfly diagram as a superposition of the 7 odd modes alone. This has been done in Fig. 3, which nicely brings out the equatorward and poleward migrations of the anti-symmetric pattern, as well as the $11 \mathrm{yr}$ polarity reversals. This is the pattern that dynamo models of the sun need to reproduce. 
Note that each linearly superposed mode is strictly sinusoidal and therefore time symmetric, whereas the composite diagram of Fig. 3 exhibits a clear arrow of time as a consequence of the definite sense of latitude migration of the pattern. As all the superposed sinusoidal modes have the same frequency, the time direction is exclusively determined by the systematic, relative phase relations between the modes of different $\ell$ values, as will be seen below.

\subsection{Amplitudes and phases, and their use for dynamo inversions}

The amplitudes and phases determined by the iterative least squares fit to the pattern of Fig. 1 are given in Fig. 4. The phases in the diagram to the lower right have been obtained from the time lags via Eq. (10), adding multiples of $2 \pi$ to some of the $\phi_{\ell}$ values to bring out the systematic shift of the phases with increasing $\ell$. It is this systematic shift that establishes the arrow of time in Fig. 3.
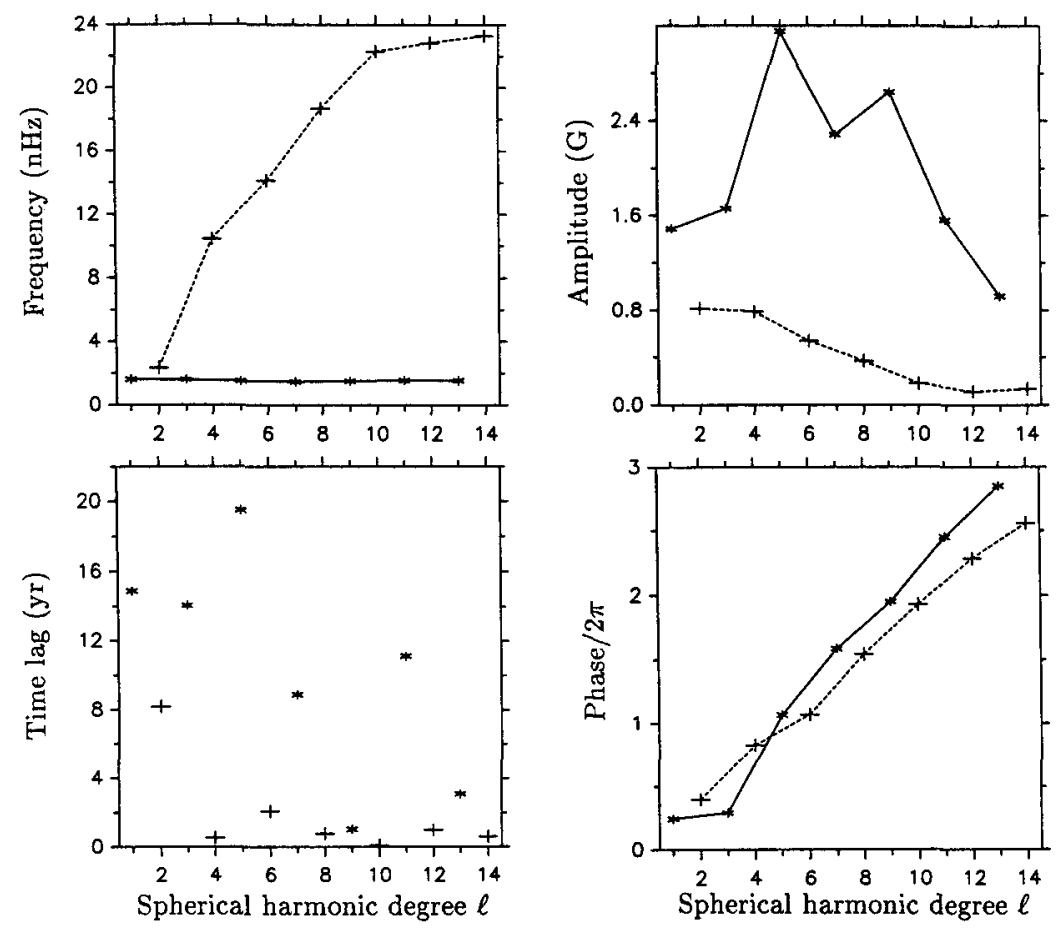

Figure 4. Frequencies, amplitudes, and phases of the odd (stars and solid lines) and even (pluses and dashed lines) modes. Adapted from Stenflo (1988).

We notice in Fig. 4 that the maximum mode amplitude does not occur for the dipole $(\ell=1)$ mode, as is often assumed, but for the mode with $\ell=5$. The even-mode amplitudes are smaller than the odd-mode ones by typically a factor of five, and the amplitude declines monotonically with $\ell$. 
The amplitudes and phases of the odd modes represent 14 observables that may be used as observed boundary constraints for inversions of the dynamo problem. Let us give an example of how such an inversion can be done. The main parameters governing the operation of the solar dynamo are the helicity, the turbulent diffusivity, and the angular velocity gradient. As the angular velocity of rotation can be obtained from helioseismology, it need not be regarded as an unknown parameter for the dynamo problem. What remains is therefore the depth and latitude variation of the helicity and diffusivity. If this variation is parameterized in terms of a sufficiently small number of free parameters, then an inversion using iterative least squares fitting of the observables will give us the values of the free model parameters with error bars.

This type of inversion approach has proven very useful even for highly nonlinear problems, e.g. the inversion of Stokes line profiles to derive the temperature stratification in the interior of small-scale magnetic fluxtubes (Keller et al., 1990). It is important to choose the free parameters of the model carefully to make the problem numerically well conditioned, and test for the uniqueness of the solution by trying iterations with widely different starting values. The goodness of the fit is given by the resulting value of $\chi^{2}$, which tells us how well the observables can be reproduced within the general framework of the chosen theoretical model.

\section{The non-axisymmetric modes}

\subsection{Effect of differential rotation}

As we cannot observe the sun simultaneously from all sides and thus not sample all longitudes simultaneously, the longitude and time coordinates are not independent of each other, but are related according to Eq. (3). This relation is somewhat arbitrary, since it depends on the choice of a rigidly rotating coordinate system, and we know that the sun in fact rotates differentially (and there is even a coexistence of different rotation laws, as we will see in Sect. 5). In contrast to the axisymmetric modes, for which the longitude information is simply averaged away, differential rotation has a profound effect on the non-axisymmetric modes (with $m \neq 0$ ).

To obtain information on possible periodicities and resonant behaviour of the non-axisymmetric modes we need to derive the Fourier transform $\tilde{c}_{\ell}^{m}(\nu)$ of the time series of the harmonic coefficients. These coefficients are formally determined by Eq. (2), which can be expressed more explicitly, using the associated Legendre polynomials $P_{\ell}^{m}(x)$, as

$$
c_{\ell}^{m}(t)=f_{\ell}^{m} \int_{-1}^{1} \mathrm{~d} x P_{\ell}^{m}(x) \int_{-\pi}^{\pi} \mathrm{d} \varphi B(x, \varphi, t) e^{-i m \varphi},
$$

where

$$
f_{\ell}^{m}=(-1)^{m} \sqrt{\frac{2 \ell+1}{4 \pi} \frac{(\ell-m) !}{(\ell+m) !}}
$$


The second integral in Eq. (11) constitutes a Fourier transform over a circular longitude interval, while the first integral makes a Legendre transform of the second integral.

Due to the coupling between time and longitude, the longitude interval of $2 \pi$ used in Eq. (12) can be regarded as a time interval of length $P_{C}$, the Carrington period. We can thus replace the Fourier transform in longitude by a Fourier transform over an infinite time interval, if we also introduce a time window function $w_{C}$ of width $P_{C}$, with a value of unity inside the window, zero outside. Accordingly

$$
c_{\ell}^{m}(t)=2 \pi \nu_{C} \int_{-\infty}^{\infty} D_{\ell}^{m}\left(t^{\prime}\right) w_{C}\left(t^{\prime}-t\right) e^{i 2 \pi m \nu_{C} t^{\prime}} \mathrm{d} t^{\prime},
$$

where

$$
D_{\ell}^{m}(t)=f_{\ell}^{m} \int_{-1}^{1} B(x, t) P_{\ell}^{m}(x) \mathrm{d} x
$$

is the Legendre transform of the radial magnetic field for a given longitude and time $t$.

The needed Fourier transform, defined by Eq. (6), of the time series described by Eq. (13), then becomes

$$
\tilde{c}_{\ell}^{m}(\nu)=2 \pi \tilde{D}_{\ell}^{m}\left(\nu-m \nu_{C}\right) \operatorname{sinc}\left(\nu / \nu_{C}\right)
$$

where the sinc function, defined by

$$
\operatorname{sinc}\left(\nu / \nu_{C}\right)=\frac{\sin \left(\pi \nu / \nu_{C}\right)}{\pi \nu / \nu_{C}},
$$

arises from Fourier transforming the window function.

We can now see how differential rotation affects the frequency spectrum of the non-axisymmetric modes. The Fourier transform $\tilde{D}_{\ell}^{m}(\nu)$ of the Legendre transform time series $D_{\ell}^{m}(t)$ is dominated by the effect of the pattern recurrence after an integer number of rotation periods. This recurrence causes "rotation peaks" in the frequency spectrum to occur at frequencies $\nu=m \nu_{R}$, where $\nu_{R}$ is the rotation frequency. $\nu_{R}$ lies in the neighbourhood of $\nu_{C}$, but it varies with latitude, due to the sun's differential rotation. The Legendre transform superposes the contributions from different latitudes with different weights. Therefore, instead of ideally having $\delta$-function peaks at $m \nu_{R}$, we get a distribution or "forest" of subpeaks around $m \nu_{C}$.

When forming the Fourier transform $\tilde{c}_{\ell}^{m}(\nu)$ of the harmonic coefficients, the "rotational forest" of the given $m$ value is translated down to the region around zero frequency, as seen by Eq. (15). The sinc function in Eq. (15) serves as a fixed low-pass filter, which transmits the low frequencies while suppressing the rotational peaks of the other, not wanted $m$ values. The problem is that the rotational distribution around zero frequency covers the whole frequency range where evolutionary effects of the non-axisymmetric modes are expected. We need to somehow dig out the subtle evolutionary information from the "rotational jungle". This is not possible by direct inspection of the power spectra as was done for the axisymmetric modes, but requires the procedure that will be described next. 


\subsection{Extraction of the "evolutionary splitting"}

Evolutionary periodicities or resonances will cause "evolutionary splitting" of the rotational peaks. The systematic splitting pattern of each peak can be extracted by making an autocorrelation analysis of the Fourier transform of the harmonic coefficients. The complex autocorrelation function is defined by

$$
A_{\ell}^{m}(\tau)=\left\langle\tilde{c}_{\ell}^{m}(\nu) \tilde{c}_{\ell}^{m *}(\nu+\tau)\right\rangle /\left\langle\left|\tilde{c}_{\ell}^{m}(\nu)\right|^{2}\right\rangle,
$$

where $\tau$ is the frequency lag.

An evolutionary resonance, like the Hale frequency $\nu_{H}=(22 \mathrm{yr})^{-1}$, gives rise to doublets with a total peak separation of $2 \nu_{H}$, which results in an autocorrelation peak at $\tau=2 \nu_{H}$. Due to this frequency doubling we will in the following diagrams give the autocorrelation results vs. $\tau / 2$ instead of vs. $\tau$, to allow us to read off the relevant frequencies directly.

The spatial resolution of our data set allows us to compute the harmonic coefficients for $m$ values from zero through 17 without aliasing. In Fig. 5 we have averaged the complex autocorrelation functions for all the $17 m$ values $1 \leq m \leq 17$ for each given, fixed value of $\ell-m$. The moduli of these averaged autocorrelation functions have then been plotted in a grey-scale representation (with darker regions representing higher autocorrelation) as functions of $\tau / 2$. As odd and even values of $\ell-m$ correspond to patterns anti-symmetric and symmetric with respect to reflections in the equatorial plane, respectively, Fig. 5 allows us to look for any possible breaking of parity symmetry, which was such a prominent feature in the case of the axisymmetric modes. We also recall that $\ell-m+1$ represents the number of zones of the associated Legendre polynomial $P_{\ell}^{m}$, so $\ell-m$ can also be regarded as representative of a zonal number. The global ( $\ell-m$-independent) grey scale cuts used in Fig. 5 will be defined in Fig. 6 below.

In contrast to the axisymmetric modes, the non-axisymmetric modes in Fig. 5 do not show any sign of parity dependence, and seem to have no $\ell-m$-dependent dispersion relation. Allowing for the noise fluctuations, the diagrams of Fig. 5 are instead characterized by horizontal bands, indicating a resonant but $\ell-m$ independent evolution of the non-axisymmetric modes. The $22 \mathrm{yr}$ resonance dominates, but the grey-scale cuts have been set low in Fig. 5 to bring out the other low-amplitude resonances. We see for instance one band with a period of 4-5 yr, with the next band occurring around the second harmonic of this frequency.

As there seems to be no $\ell$ and $m$ dependence of the resonant pattern, we may average the complex autocorrelation functions for all the spatially resolved $\ell$ and $m$ modes to better bring out the low-amplitude resonances from the background noise. This has been done separately for odd and even parity $(\ell-m)$ in Fig. 6 . Thus $119(=7 \times 17)$ autocorrelation functions contribute to the odd mode curve, $136(=8 \times 17)$ functions to the even mode curve. The right diagram of Fig. 6 gives a close-up look at the lower-frequency region of the left diagram. 


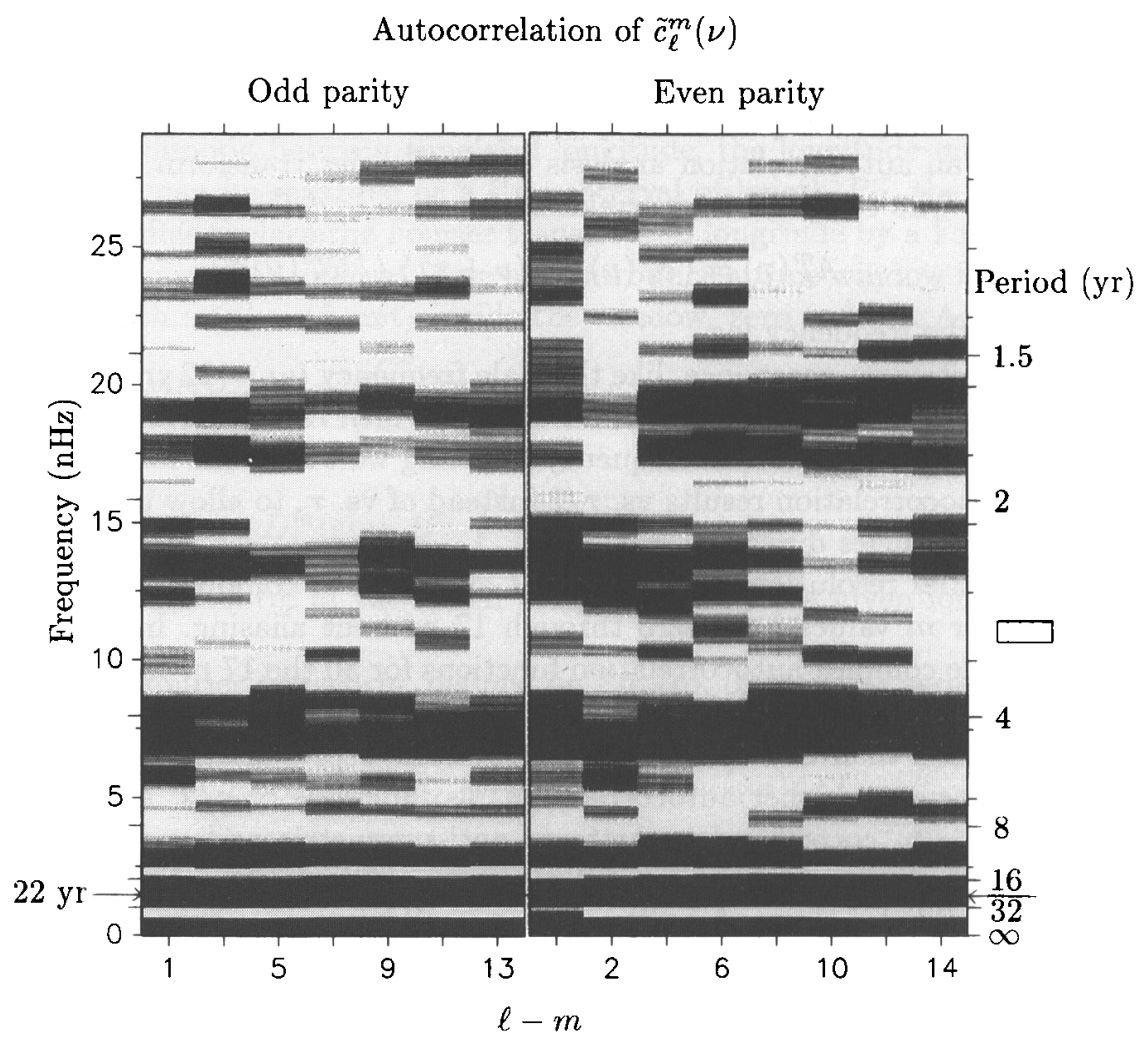

Figure 5. Autocorrelation amplitudes of the non-axisymmetric modes, as functions of frequency $(\tau / 2)$ and zonal number. The results are given in separate diagrams for odd and even $\ell-m$, and contain the averaged contributions from all the $m$ values from 1 through 17. Global grey-scale cuts, independent of the value of $\ell-m$, have been used (as defined in Fig. 6). From Stenflo and Güdel (1988).

\subsection{Interpretation of the non-axisymmetric modes}

The most striking feature of Fig. 6 is the almost perfect agreement between the independently derived curves representing odd and even parity. The amplitudes of the dominating $22 \mathrm{yr}$ peak are for instance equal to within $2 \%$. Even the highfrequency, low-amplitude wiggles are extremely well matched between the two parities. This strongly suggests that practically all of these wiggles are real and of solar origin, and may therefore be subject to a serious analysis.

The horizontal band with its second harmonic seen in Fig. 5 appears in Fig. 6 with an apparent triplet structure, with a central frequency of 4.6 or $2.3 \mathrm{yr}$, surrounded by side peaks shifted by $\pm(22 \mathrm{yr})^{-1}$. At higher frequencies the diagram gets very crowded with apparently overlapping peak patterns. This part of the diagram cannot be interpreted without a much more elaborate analysis, which has not yet been carried out. 

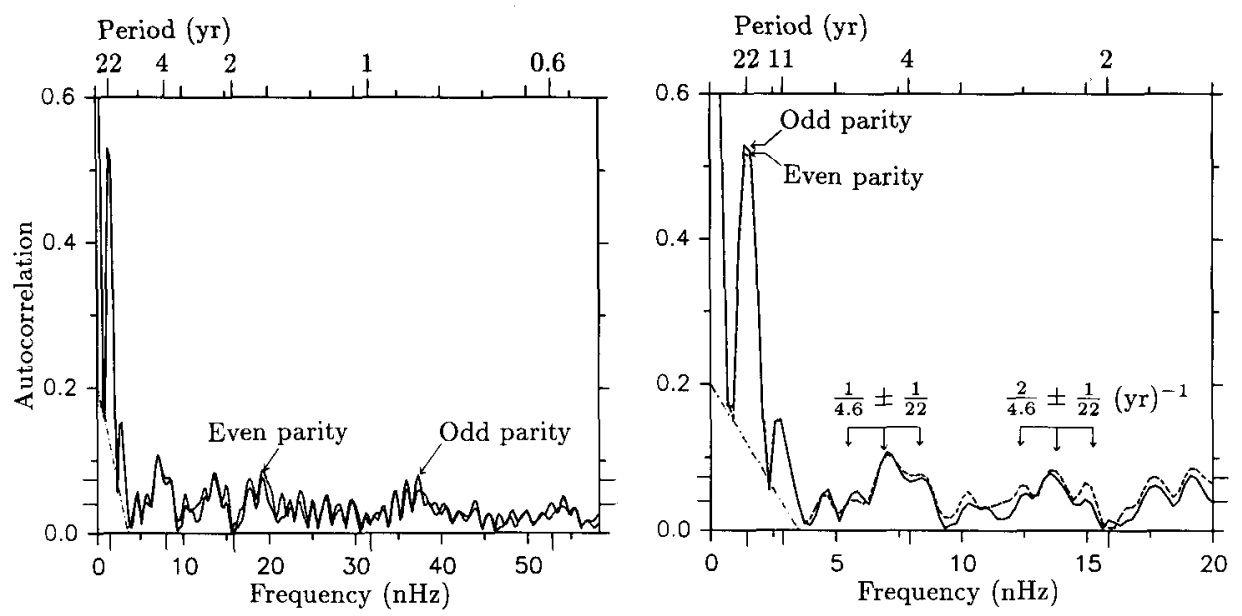

Figure 6. Autocorrelation amplitude as a function of frequency $\tau / 2$, for odd (solid curve) and even (dashed curve) values of $\ell-m$. The curves represent averages of the contributions from all the $\ell$ and $m$ values with the given parity. A possible triplet structure with its second harmonic is indicated in the right diagram. The cuts used for the grey scale of Fig. 5 are marked by the two longest tick-marks on the vertical axis. From the lower to the upper tick-mark the grey scale varies from white to black. Before applying the cuts, however, the lower, straight-line envelop marked by the dashed-dotted line in the lower left-hand corner has been subtracted, to bring the minima surrounding the $22 \mathrm{yr}$ peak down to the range of the cuts. From Stenflo and Güdel (1988).

The following scenario may provide a general, qualitative framework for understanding the results for the non-axisymmetric modes. The dominant $22 \mathrm{yr}$ mode represents the periodicity in the dynamo production of new toroidal flux inside the sun. This flux is made visible when part of it emerges to the surface from the production region. Flux emergence is triggered by non-axisymmetric deformations of the toroidal flux ropes. As these deformations may propagate along the closed toroidal flux as waves, they may develop a resonant structure through constructive and destructive interference. These interferences will then appear as periodicities in the emergence of new bipolar flux and get revealed by our analysis. The frequencies that we find then represent the eigenmodes of the underlying waves. Wave periods of the order of a few years are expected if the waves travel with the Alfvén velocity along $\mathrm{kG}$ fields at the bottom of the convection zone.

When a localized bipolar magnetic region is described by an expansion in terms of spherical harmonics, it will contribute to the harmonic modes of all $\ell$ and $m$ values up to a spatial frequency corresponding to the inverse size of the bipolar region. Therefore the periodicities in the flux emergence will appear with the same 
frequencies for all $\ell$ and $m$ values, which explains the horizontal band structure in Fig. 5. The available amount of flux is modulated by dynamo action with a $22 \mathrm{yr}$ period, and this modulation combines with the various frequencies of flux emergence to produce the triplet splitting patterns seen in Fig. 6 (Stenflo and Güdel, 1988).

If this general scenario is adopted, then the various low-amplitude resonant frequencies may be used to provide diagnostic constraints on the distribution of magnetic flux and on the field strengths in the solar interior.

\section{Pattern phase velocity and the internal angular velocity}

\subsection{Coexistence of two magnetic field rotation laws}

The observed pattern of surface magnetic flux may be used as a tracer for determinations of the sun's differential rotation. Two different methods have been used: (1) The "longitude displacement method" (Snodgrass, 1983), and (2) the "pattern recurrence method" (Stenflo, 1989). Although the two methods have been applied to the same magnetograph data, and both measure the phase velocity of the magnetic pattern, they produce entirely different rotation laws.

The first method determines the proper motion in the longitude direction of the pattern by comparing consecutive full-disk magnetograms, separated in time by 1-4 days. The longitude displacement of the pattern is determined by crosscorrelation of the flux pattern in the different magnetograms, without any identification of individual flux features. The second method uses only the flux sampled at the central meridian. For each latitude zone we get the field as a function of time, and the time series that has been used is $26 \mathrm{yr}$ long (1959-1985), as described above in Sect. 2. Autocorrelation or power spectrum analysis can be directly applied to the $26 \mathrm{yr}$ time series for each of the 30 latitude zones. The recurrence of the pattern at the central meridian after an integer number of solar rotations gives rise to equidistant peaks in the autocorrelation function as well as in the power spectrum. The peak locations determine the pattern phase velocity.

Figure 7 compares the resulting rotation laws. The four different types of symbols plotted refer to rotation rates determined from the center of gravity of each of the four first autocorrelation peaks (with lags from one to four months). They show that the four peaks give consistently very similar rotation rates. The synodic rotation period reaches a maximum of about 29.5 days around a latitude of about $55^{\circ}$, with a tendency for a small polar spin-up. In the case of the Snodgrass law (solid curve), however, the rotation period increases steeply with latitude, reaching a value of about 38 days at the poles. The Snodgrass law on the other hand closely agrees with the rotation law determined from Doppler measurements (Howard et al., 1983), represented by the dashed-dott ed curve. 


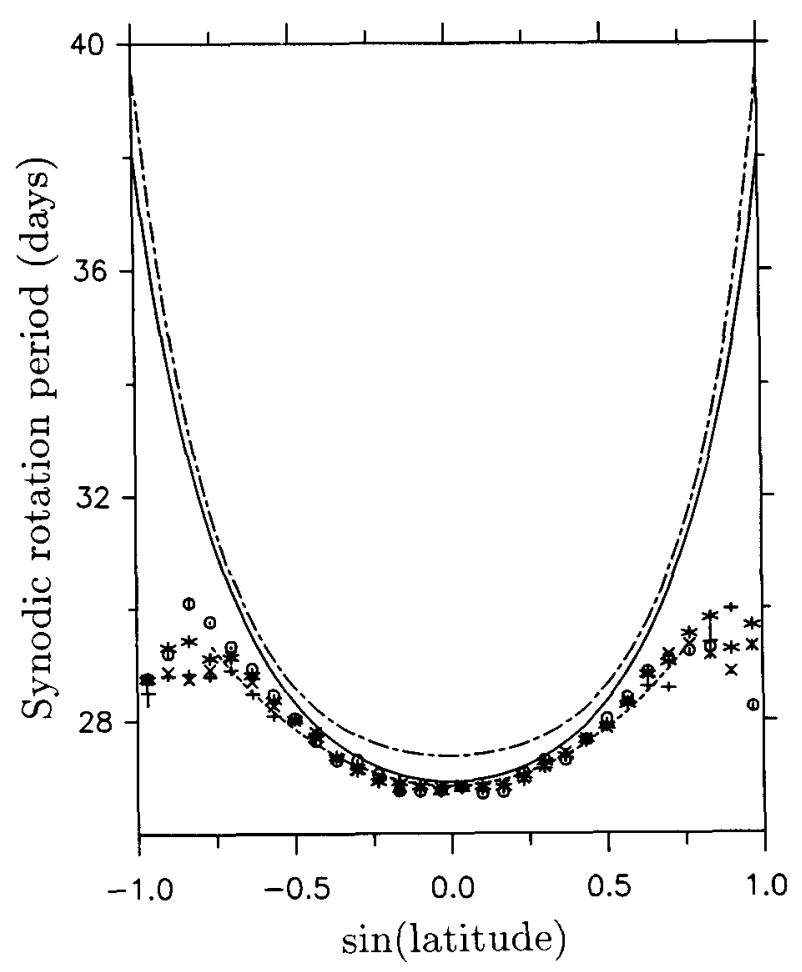

Figure 7. Synodic period of rotation of the magnetic field pattern. The four different symbols represent the rotation rate determined with the recurrence method, using lags of one (open circles), two (stars), three (pluses), and four (crosses) rotation periods. The solid curve represents the rotation law of Snodgrass (1983), while the dashed-dotted curve has been obtained from Doppler line shifts (Howard et al., 1983). From Stenflo (1989).

\section{2 "Active longitudes" and pattern regeneration}

We note that the longitude displacement and recurrence methods both refer to the phase velocity of the magnetic pattern. Both use a rather coarse spatial grid (Snodgrass averages over about one arcmin square). The only essential difference between them is the time lag used in the correlation analysis, 1-4 days for the longitude displacement method, 27 days or more for the recurrence method.

The close agreement between the longitude displacement and the Doppler results shows that the pattern phase velocity determined from small correlation lags agrees with the plasma velocity in the photosphere. It has in the past been popular to invoke surface flux redistribution processes (like turbulent diffusion and meridional circulation) to explain how the phase velocity will deviate with time more and more from the plasma velocity, such that a quasi-rigid pattern rotation is approached (Sheeley et al., 1987). Such processes can however be ruled out as an explanation of the two coexisting rotation laws, even if the time scale for such 
a redistribution would be arbitrarily short, e.g. less than a month (Stenflo, 1989). The reason is the following:

In the redistribution models (e.g. Sheeley et al., 1987) a quasi-rigid pattern phase velocity develops due to reshuffling of existing, old surface flux. To clarify the consequences of such models, let us assume that the flux pattern initially rotates with the plasma velocity but within some short time, say a month, develops a quasi-rigid phase velocity. If then the longitude displacement method of Snodgrass is applied, this quasi-rigid pattern phase velocity will be picked up, in strong contradiction with what is being observed for the real sun. Once a quasi-rigid pattern phase velocity has been established by reshuffling of the old flux, assuming no significant contributions from new emerging flux, then correlation analysis in the limit of small lags, like the longitude displacement method, will find the quasirigid phase velocity, not the plasma velocity. In contrast Snodgrass (1983) finds a steep differential rotation law that agrees with the plasma rotation. This law is moreover found to be time invariant, applying to the whole $15 \mathrm{yr}$ period that he studied.

The only process that has been found to account for the coexistence of the two pattern phase velocities is pattern renewal by flux emergence from the solar interior over a time scale of less than 27 days, but more than a few days. In this scenario the surface magnetic pattern observed at any given time represents new flux that has emerged within the past month, and does not consist of old fluxes that have their origin in redistribution of active-region fluxes. New flux that arrives at the surface will initially drift with the "local winds", and thus have a phase velocity that agrees with the plasma velocity, as found by the longitude displacement method. As the flux turnover time is less than a month, however, the recurrence method will not measure something related to the photospheric plasma velocity, but will correlate the regions of flux emergence, the "active longitudes", with each other. They rotate quasi-rigidly, not because of some redistribution processes, but because of the rotation properties of the source in the solar interior, from which the surface pattern is constantly being replenished.

This explanation revives and generalizes the old concept of "active longitudes" (cf. Sawyer, 1968; Bogart, 1982). The concept is generalized because it is used here not so much for active-region flux, but primarily for flux emerging far from the active-region belts, at high latitudes (where the discrepancy between the two rotation laws is the most pronounced). It may appear surprising that flux emergence would be so pervasive and occur at such an enormous rate. Video magnetograph observations of flux emergence and cancellation rates (Martin, 1990) however indicate that the turnover time scale for the flux pattern is really this extremely short. No other viable explanation of the two coexisting rotation laws has been proposed. 


\subsection{Location of the magnetic source region inside the sun}

The recurrence method has also been used to explore if the quasi-rigid rotation law varies with the phase of the $11 \mathrm{yr}$ cycle. Such variations could occur if for instance the depth distribution of the sources of magnetic flux varies with the cycle (and the angular velocity of rotation varies with depth), or if there are torsional oscillations in the source region. For this purpose the $26 \mathrm{yr}$ time series have been divided into 21 time windows of length 16 Carrington rotations $(\approx 1.2 \mathrm{yr})$, and the rotation rate has been determined by power spectrum analysis for each of the $21 \times 30=630$ time series.

The quasi-rigid rotation law determined by the recurrence method is thereby found to be time invariant within tight limits (Stenflo, 1990). Thus the random rms fluctuations are $\lessgtr 0.3 \%$ at low latitudes, increasing to $2-3 \%$ at high latitudes (where the signal-to-noise ratio is smaller due to the smaller amplitudes of the lineof-sight magnetic flux there). Translated to linear velocities for a source region at the bottom of the convection zone these limits correspond to $\$ 4 \mathrm{~m} \mathrm{~s}^{-1}$ at low latitudes, $\lesssim 10-20 \mathrm{~m} \mathrm{~s}^{-1}$ at high latitudes. In contrast the Snodgrass rotation law deviates from the recurrence rotation law by as much as about $20 \%$ near the poles.

These results speak in favour of flux storage at the bottom of the convection zone throughout the course of the solar cycle. If the storage depth would vary with the cycle, then any radial gradient of the angular velocity of rotation would show up as an apparent cycle-dependent variation of the phase velocity of the surface pattern, which is not observed. Now results from helioseismology (Morrow, 1988; Libbrecht, 1988; Brown et al., 1989; Dziembowski et al., 1989) suggest that the radia 1 gradient of the angular velocity is small throughout the bulk of the convection zone, but that there is a transition to a rigidly rotating core near the bottom of the convection zone. As however the bulk of the convection zone rotates nearly with the surface rate according to this picture, the flux storage region with its quasi-rigid rotation can hardly be located there. Thus the results from helioseismology when combined with the magnetic-field data support the conclusion that the magnetic flux is stored near the bottom of the convection zone. Flux storage at this location, which has been advocated in the past on theoretical grounds (e.g. Spiegel and Weiss, 1980; Spruit and van Ballegooijen, 1982; DeLuca, 1987; Schüssler, 1987), has thus been given empirical support.

\subsection{Angular velocity structure of the solar interior}

The apparent absence of time variations in the observed phase velocity of the source pattern is easier to understand if the phase velocity approximately agrees with the plasma velocity in the source region. Any deviation between the phase and plasma velocities would be expected to vary with the solar cycle, since the dynamo evolves with time, and the sources of flux migrate in latitude. The absence of observed variations suggests that the phase velocity closely follows the plasma velocity, which should be time invariant (as long as the kinetic energy densities at the bottom of the convection zone are much larger than the magnetic energy densities, which is the case if the field strengths are much less than megagauss). 
It thus appears that the longitude displacement method of Snodgrass gives the rotation law for the surface layers (the photosphere), whereas the recurrence method gives the law for the source region, which has a likely location at the bottom of the convection zone. Our analysis of the magnetic-field data however has not provided us with any information on the angular velocity of the intervening layers of the convection zone. If we use the mathematically simplest procedure (without any physical justification) of linear interpolation between these two regions (top and bottom of the convection zone), then we obtain the isocontour plot of the angular-velocity structure of the sun given to the right in Fig. 8. It is compared with the rotational structure obtained from helioseismology (Libbrecht, 1988), given to the left in Fig. 8. Such a comparison has also been provided by Brandenburg (1990).
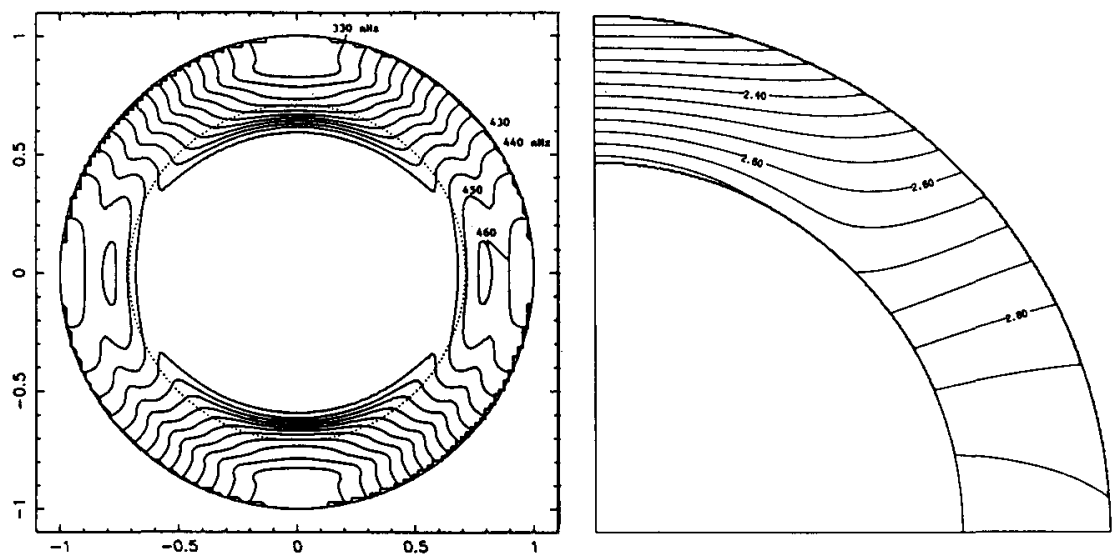

Figure 8. Isocontours of the sun's angular velocity of rotation, derived from helioseismology (left diagram, from Libbrecht (1988)) and from the observed pattern of surface magnetic fields (right diagram, from Stenflo (1989)). The two rotation laws for the pattern phase velocity have been taken to represent the top and bottom of the convection zone, with linear interpolation in between.

In spite of the simplistic use of linear interpolation between the top and bottom of the convection zone there are considerable similarities between the two diagrams of Fig. 8. Thus while the contours are predominantly radial at low latitudes, they become increasingly perpendicular to the sun's rotational axis at higher latitudes. This qualitative result is in direct contradiction with predictions from numerical simulations that the contours should be largely parallel to the rotational axis (Glatzmaier, 1987), but it agrees with other theories of the sun's differential rotation (e.g. Rüdiger and Tuominen, 1990).

Closer agreement with the helioseismology diagram would be obtained if we instead of linear interpolation between the top and bottom of the convection zone would let the surface rotation law prevail throughout most of the convection zone, and do our interpolation within a transition zone near the bottom of the convection 
zone. This implies that the isocontours in the right diagram of Fig. 8 would be strictly radial from the surface down to the top of the transition zone, and that the isocontour

structure within the transition zone would look like a compressed version of the present right-hand diagram.

The only significant disagreement with helioseismology occurs at low latitudes, in the active-region belts, where helioseismology finds an angular velocity at the bottom of the convection zone that is slower as compared with the surface rate, whereas our low-latitude rates instead are very similar to the surface rates, with a tendency of being slightly faster (although by less than one percent). The cause for this discrepancy has not been identified yet, but it may be that the scenario of rapid regeneration of the flux pattern over a time scale of less than a month

is less applicable at active region-latitudes, since many of the individual flux elements (e.g. sunspots) there are much larger than at high latitudes and have much longer lifetimes, which means that their old fluxes will dominate the pattern for much longer periods than the flux elements at high latitudes.

The regeneration scenario was introduced as the necessary and apparently only possibility for explaining the coexistence of highly different pattern phase velocities. The two rotation laws rapidly diverge from each other at high latitudes, but at low latitudes the difference is below one percent and not very significant. Therefore, if the low latitudes were considered in isolation, there would be practically only one single magnetic rotation law, and thus no problem requiring an explanation.

These questions may be clarified, on the one hand by direct observations of the flux regeneration rate as a function of latitude, on the other hand by more definite observations of the p-mode rotational splittings. The regeneration problem is particularly difficult, since most of the "background" magnetic flux far from active regions is expected to emerge and disappear over very small spatial scales, possibly beyond the resolution capabilities of currently used magnetographs. It is the accumulated effect of many such small-scale emergence and disappearance events that produces the large-scale flux pattern that we use for our correlation analyses. The key to an understanding of the large scales thus lies in the observation of the smallest scales, which requires powerful telescopes. The planned, international LEST (Large Earth-based Solar Telescope) has a design (Engvold and Andersen, 1990) that is optimized for solving this problem.

Acknowledgements. The Mount Wilson data used throughout this review has been recorded under the direction of Robert Howard and been subsequently organized in the $30 \times 36$ array synoptic format at the High Altitude Observatory. The Kitt Peak synoptic data set has been compiled and made available by J.W. Harvey. 


\section{References}

Bogart, R.S.: 1982, Solar Phys. 76, 155

Brandenburg, A.: 1990, Ph.D. Thesis, Observatory and Astrophysics Laboratory Rep. 2/1990, Univ. of Helsinki

Brown, T.M., Christensen-Dalsgaard, J., Dziembowski, W.A., Goode, P., Gough, D.O., Morrow, C.A.: 1989, Astrophys. J. 343, 526

DeLuca, E.E.: 1987, Ph.D. Thesis, Univ. of Colorado (available as NCAR Cooperative Thesis No. 104)

Dziembowski, W.A., Goode, P.R., Libbrecht, K.G.: 1989, Astrophys. J. 337, L53

Engvold, O., Andersen, T. (eds.): 1990, LEST Design, LEST Foundation

Glatzmaier, G.A.: 1987, in The Internal Solar Angular Velocity, eds. B.R. Durney, S.Sofia, Astrophys. Space Sci. Library 137, 263

Gokhale, M.H., Javaraiah, J.: 1989, Monthly Not. Royal Astron. Soc., in press

Gokhale, M.H., Javaraiah, J., Hiremath, K.M.: 1990, in Solar Photosphere: Structure, Convection, and Magnetic Fields, ed. J.O. Stenflo, IAU Symp. 138, 375

Howard, R., Adkins, J.M., Boyden, J.E., Cragg, T.A., Gregory, T.S., LaBonte, B.J., Padilla, S.P., Webster, L.: 1983, Solar Phys. 83, 321

Hoyng, P.: 1987, Astron. Astrophys. 171, 357

Hoyng, P.: 1988, Astrophys. J. 332, 857

Hoyng, P.: 1990, in Solar Photosphere: Structure, Convection, and Magnetic Fields, ed. J.O. Stenflo, IAU Symp. 138, 359

Keller, C.U., Solanki, S.K., Steiner, O., Stenflo, J.O.: 1990, Astron. Astrophys. 233, 583

Libbrecht, K.G.: 1988, in Seismology of the Sun and Sun-Like Stars, eds. V. Domingo, E.J. Rolfe, ESA SP-286, p. 131

Martin, S.F.: 1990, in Solar Photosphere: Structure, Convection, and Magnetic Fields, ed. J.O. Stenflo, IAU Symp. 138, 129

Morrow, C.A.: 1988, Ph.D. Thesis, Univ. of Colorado (available as NCAR Cooperative Thesis No. 116)

Rüdiger, G., Tuominen, I.: 1990, in Solar Photosphere: Structure, Convection, and Magnetic Fields, ed. J.O. Stenflo, IAU Symp. 138, 315

Sawyer, C.: 1968, Ann. Rev. Astron. Astrophys. 6, 115

Schüssler, M.: 1987, in The Internal Solar Angular Velocity, eds. B.R. Durney, S. Sofia, Astrophys. Space Sci. Library 137, 303

Sheeley, N.R., Jr., Nash, A.G., Wang, Y.-M.: 1987, Astrophys. J. 319, 481

Snodgrass, H.: 1983, Astrophys. J. 270, 288

Spiegel, E.A., Weiss, N.O.: 1980, Nature 287, 616

Spruit, H.C., van Ballegooijen, A.A.: 1982, Astron. Astrophys. 106, 58

Stenflo, J.O.: 1988, Astrophys. Space Sci. 144, 321

Stenflo, J.O.: 1989, Astron. Astrophys. 210, 403

Stenflo, J.O.: 1990, Astron. Astrophys. 233, 220

Stenflo, J.O., Güdel, M.: 1988, Astron. Astrophys. 191, 137

Stenflo, J.O., Vogel, M.: 1986, Nature 319, 285 